\title{
Effect of Gegen Qinlian Decoction on Cardiac Gene Expression in Diabetic Mice
}

\author{
Jing Han, ${ }^{1}$ Zhenglin Wang, ${ }^{2}$ Wei Xing, ${ }^{2}$ Yueying Yuan, ${ }^{1}$ Yi Zhang, ${ }^{3}$ Tiantian Lv, ${ }^{2}$ \\ Hongliang Wang, ${ }^{2}$ Yonggang Liu, ${ }^{4}$ and ${\text { Yan } W u^{1}}^{1}$ \\ ${ }^{1}$ Institute of Chinese Medicine, Beijing University of Chinese Medicine, Beijing 100029, China \\ ${ }^{2}$ College of Basic Medicine, Key Laboratory of Ministry of Education (Syndromes and Formulas), \\ Key Laboratory of Beijing (Syndromes and Formulas), Beijing University of Chinese Medicine, Beijing 100029, China \\ ${ }^{3}$ Modern Research Center for Traditional Chinese Medicine, Beijing University of Chinese Medicine, Beijing 100029, China \\ ${ }^{4}$ College of Chinese Medicine, Beijing University of Chinese Medicine, Beijing 100029, China
}

Correspondence should be addressed to Yan Wu; nayattmm@vip.sina.com

Received 13 July 2017; Revised 30 September 2017; Accepted 26 October 2017; Published 12 December 2017

Academic Editor: Qin Feng

Copyright @ 2017 Jing Han et al. This is an open access article distributed under the Creative Commons Attribution License, which permits unrestricted use, distribution, and reproduction in any medium, provided the original work is properly cited.

\begin{abstract}
The aim of this research is to investigate the therapeutic effect of GGQL decoction on cardiac dysfunction and elucidate the pharmacological mechanisms. db/db mice were divided into DB group or GGQL group, and WT mice were used as control. All mice were accessed by echocardiography. And the total RNA of LV tissue samples was sequenced, then differential expression genes were analyzed. The RNA-seq results were validated by the results of RT-qPCR of 4 genes identified as differentially expressed. The content of pyruvate and ceramide in myocardial tissue was also measured. The results showed that GGQL decoction could significantly improve the diastolic dysfunction, increase the content of pyruvate, and had the trend to reduce the ceramide content. The results of RNA-seq showed that 2958 genes were differentially expressed when comparing the DB group with the WT group. Among them, compared with the DB group, 26 genes were differentially regulated in the GGQL group. The expression results of 4 genes were consistent with the RNA-seq results. Our study reveals that GGQL decoction has a therapeutic effect on diastolic dysfunction of the left ventricular and the effect may be related to its role in promoting myocardial glycolysis and decreasing the content of ceramide.
\end{abstract}

\section{Introduction}

There is a dramatically increasing epidemic of DM patients. The prevalence of DM is $4 \%$ in 1995 , and this number is anticipated to reach $5.4 \%$ in 2025 , amounting to 300 million DM patients [1]. Diabetic cardiomyopathy (DCM) is a major complication of diabetes, afflicting $12 \%$ of patients. The prevalence rate will reach $22 \%$ in people aged $>64$ years old [2].

A very significant aspect in early period of DCM is left ventricular (LV) dysfunction, especially diastolic dysfunction. And it is characterized by LV hypertrophy and increased cardiac fibrosis [3].

Despite of intensive glycemic, lipidemic control and neurohormonal antagonists, the progress of DCM in diabetic patients has not impeded. Even worse, cardiovascular mortality has increased due to hypoglycemia [4]. In brief, the therapeutic rules for DCM come from the treatment of heart failure and DM. No specific medications for this disease have been put to clinical use. Therefore, more studies are required to explore new agents for this complex syndrome.

Gegen Qinlian (GGQL) decoction, which is composed of Radix Puerariae (ge gen), Radix Scutellariae (huang qin), Coptis chinensis Franch (huang lian), and Radix Glycyrrhizae Praeparata (zhi gan cao), has been addressed for its medicinal effect against DM for almost ten years [5]. It has been reported that GGQL decoction has the ability to improve hyperglycemia and hyperlipidemia [6, 7]. In addition, GGQL decoction has a positive inotropic, negative frequency effect on the isolated perfused rat heart [8]. Furthermore, R Scutellariae baicalensis reduced myocardial 
infarct size in myocardial ischemia-reperfusion injured rats [9]. Berberine, a compound from C. chinensis, has negative chronotropic, positive inotropic, antiarrhythmic, and vasodilator properties [10]. Thereafter, we speculate that GGQL decoction will have protective effect against DCM.

In recent years, transcriptomics has been used to determine the mechanism of traditional Chinese medicine [11]. Thus, the purpose of this study is to evaluate the effect of GGQL decoction on DCM and elucidate the pharmacological mechanisms by transcriptomics. First, we assessed the efficacy of GGQL decoction against injured cardiac functions using the DM mouse model. Then, we evaluate the influence of GGQL decoction on changes of transcriptomics. Some of the differently expressed genes were confirmed by RT-qPCR. Our extensive studies will determine the potential targets of GGQL decoction and provide new treatment strategies for DCM.

\section{Materials and Methods}

2.1. Preparation of Gegen Qinlian Decoction. To prepare the aqueous extract of Gegen Qinlian decoction, firstly, Radix Puerariae, Radix Scutellariae, Coptis chinensis Franch, and Radix Glycyrrhizae Praeparata, at the rate of $5: 3: 3: 2$, were soaked in 10 times of distilled water $(\mathrm{v} / \mathrm{w})$ for 30 minutes and then boiled for $1 \mathrm{~h}$. The decoction was filtered and collected. Secondly, the residue was added into 10 times of distilled water (v/w) and boiled for $1 \mathrm{~h}$, and the hot decoction was filtered. Thirdly, the filtrate was mixed and concentrated to the aqueous extract.

2.2. Animals and Grouping. Studies were performed following the Guide for the Care and Use of Laboratory Animals published by the National Institutes of Health and with the permission of the Care Committee of Beijing University of Chinese Medicine. Male C57BL/KsJ db/db mice and their similar genetic background age-matched C57BL/KsJ wildtype (WT) mice were obtained from the Nanjing Biomedical Research Institute of Nanjing University (Jiangsu, China). Mice were kept in the animal house with a $12: 12 \mathrm{~h}$ lightdark cycle and controlled temperature of $22-25^{\circ} \mathrm{C}$. At 8 weeks of age, $\mathrm{db} / \mathrm{db}$ mice were randomly divided into the DB group or the GGQL group, and the age-matched WT mice were used as control. The aqueous extract of GGQL decoction was dissolved in distilled water, and the GGQL group was intragastrically administered at a dosage of $23.4 \mathrm{~g}$ crude drugs $/ \mathrm{kg} / \mathrm{d}$ for 8 weeks. The DB group and WT group were treated with an equal volume of distilled water. After 8 weeks' administration, all the mice were weighted and the blood glucose was measured. Then, all the mice were sacrificed, and the hearts were harvested. And the tissue of the left ventricle was collected and stored in the liquid nitrogen for subsequent mRNA isolation.

2.3. Echocardiographic Assessment. At the 8th week of administration, the mice were accessed by echocardiography. All the mice were taped on the heated procedure board and anesthetized with $1.5 \%$ isoflurane in oxygen. In the apical four-chamber view, the peak velocity of early diastolic mitral
TABLE 1: List of primers used in RT-qPCR.

\begin{tabular}{lc}
\hline Gene & Primer sequence $\left(5^{\prime}-3^{\prime}\right)$ \\
\hline \multirow{2}{*}{ Pgam1 } & F:AATTCAGGGAGGAACTGTGCT \\
& R:GGACAGGTTCCAGGGACAAAA \\
\hline \multirow{2}{*}{ Acer2 } & F:GCTCTGTGAAAATACTGCCACC \\
& R:CAGTGTTGGCTCTGGGTAGG \\
\hline \multirow{2}{*}{ Slc38a2 } & F:CAAACCTCCTGTGAGGGAGC \\
\hline \multirow{2}{*}{ Ppp1r3c } & F:GAATTGAGGTGACGGGACAGT \\
\hline
\end{tabular}

F: forward; R: reverse.

inflow velocity $(E)$, the peak value of late diastolic mitral inflow velocity $(A)$, and the E-to-A ratio $(E / A)$ were measured by pulsed Doppler mode, using Vevo 2100 Imaging System (VisualSonics, Canada) with a $30 \mathrm{MHz}$ high-frequency transducer. In tissue Doppler mode, the early diastolic velocity $\left(E^{\prime}\right)$ and the late diastolic velocity $\left(A^{\prime}\right)$ were measured, and ratio of early to late diastolic velocities $\left(E^{\prime} / A^{\prime}\right)$ was calculated. Data analysis was performed with the use of Vevo 2100 Analytic Software (VisualSonics, Canada).

2.4. RNA Isolation and Quality Control. Total RNA was isolated from $15 \mathrm{LV}$ tissue samples (5 samples each group) using Trizol reagent (Invitrogen, CA). Agarose gel electrophoresis was used to analyze RNA integrity and the presence of DNA contamination of samples. RNA purity (ratio of OD260/280 and OD260/230) was measured by NanoPhotometer spectrophotometer. RNA concentration was accurately quantified by Qubit 2.0 Fluorometer. And RNA integrity was accurately detected by Agilent 2100 bioanalyzer. Then, poly-A tail mRNA was enriched with Oligo(dT) beads, and the enriched mRNA was randomly fragmented by divalent cations in NEB Fragmentation Buffer. The fragmented mRNA was reverse transcripted, and the cDNA was synthesized. After end repair and addition of A tail and adaptor to the purified cDNA, cDNAs were selected about 200 bp with AMPure XP beads, PCR amplification was performed, and then the PCR product was purified. The insert size of library was detected by Agilent 2100 bioanalyzer, and the effective concentration of the library was accurately quantified.

2.5. RNA Sequencing. The mRNA was sequenced with Illumina HiSeq2500 platform. The read numbers mapped of each gene were counted with HTSeq v0.6.1. RPKM (reads per kilobase of exon model per million mapped reads) of each gene was calculated on the basis of the gene length and reads count mapped to the gene.

Gene Ontology (GO) and Kyoto Encyclopedia of Genes and Genomes (KEGG) enrichment was applied by the GOseq $\mathrm{R}$ package and KOBAS. The results of clustered analysis of differentially expressed genes were showed with the heatmap.

2.6. RT-qPCR Validation of RNA-seq. Four genes identified as differentially expressed were randomly selected to validate 


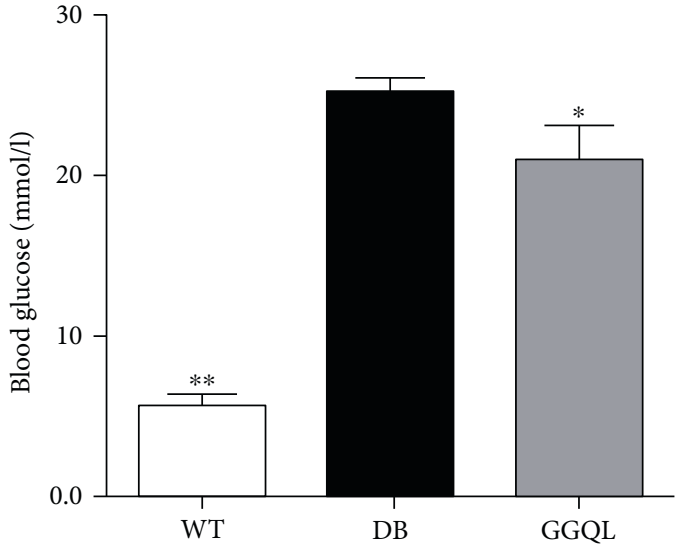

(a)

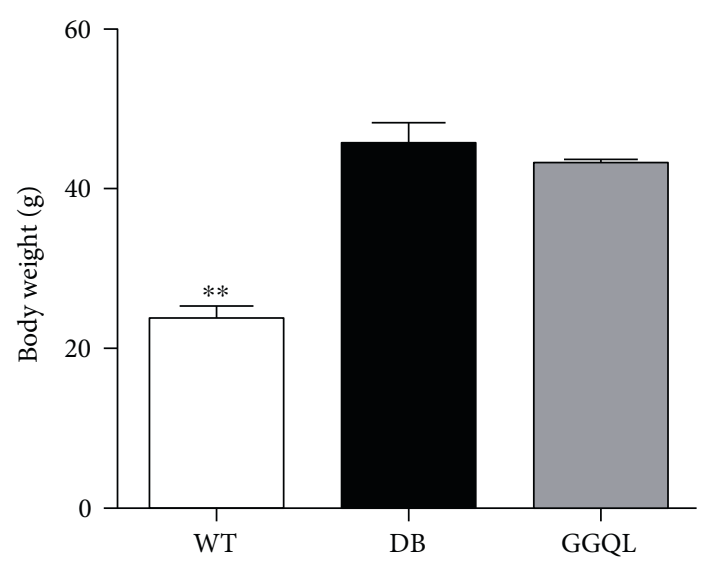

(b)

Figure 1: The results of blood glucose and bodyweight. (a) Blood glucose and (b) bodyweight in each group $(n=8)$. The DB group was the reference group to calculate $P$ values, ${ }^{*} P<0.05$ and ${ }^{* *} P<0.01$.

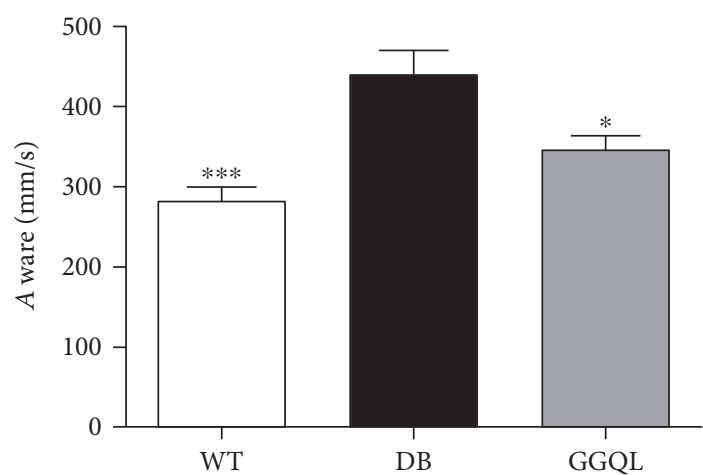

(a)

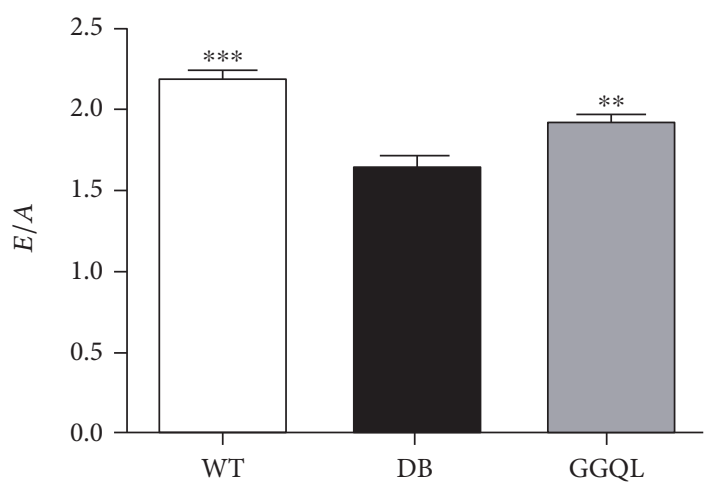

(c)

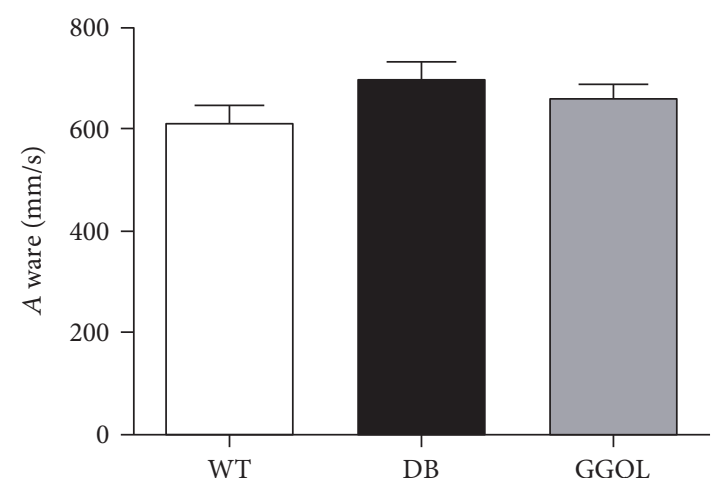

(b)

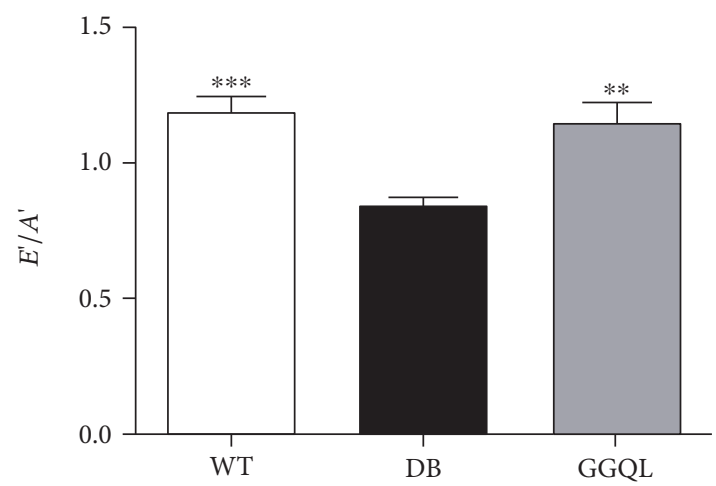

(d)

Figure 2: The results of cardiac function detected by echocardiograph. (a) $E$ wave, (b) $A$ wave, (c) $E / A$ ratio, and (d) $E$ ' $A^{\prime}$ ratio in each group $(n=9)$. The DB group was the reference group to calculate $P$ values, ${ }^{*} P<0.05,{ }^{* *} P<0.01$ and ${ }^{* * *} P<0.001$.

the results of RNA-seq by RT-qPCR. $1 \mu \mathrm{g}$ of total RNA was reverse transcribed to $\mathrm{CDNA}$ in $20 \mu \mathrm{l}$ reaction. Custom gene-specific primers for qRT-PCR were designed by Primer-BLAST, and the sequences of primers are listed in Table 1 . Gene expression was measured in triplicates using the ABI StepOnePlus ${ }^{\circledR}$ instrument (ABI, USA) and SYBR GreenImaster mix (Roche, USA). The protocol of the reactions was $2 \mathrm{~min}$ at $50^{\circ} \mathrm{C}$ and $10 \mathrm{~min}$ at $95^{\circ} \mathrm{C}$, followed by 40 cycles of denaturation at $95^{\circ} \mathrm{C}$ for $15 \mathrm{~s}$ and annealing at the corresponding melting temperatures for $30 \mathrm{~s}$, and the melt curve was detected from $60^{\circ} \mathrm{C}$ to $95^{\circ} \mathrm{C}\left(0.5^{\circ} \mathrm{C}\right.$ increments every $5 \mathrm{~s}$ ). All the expression levels of mRNA were normalized with GAPDH as a housekeeping gene.

2.7. Measurement of Ceramide in Myocardial Tissue. On the day of extraction, the myocardial tissues were weighed 
Cluster analysis of differentially expressed genes

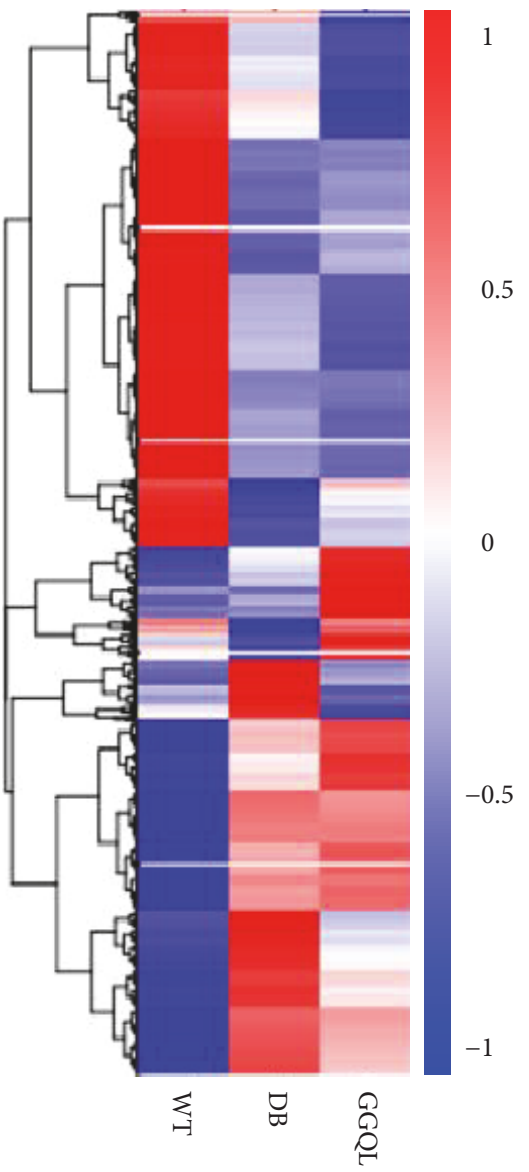

(a)

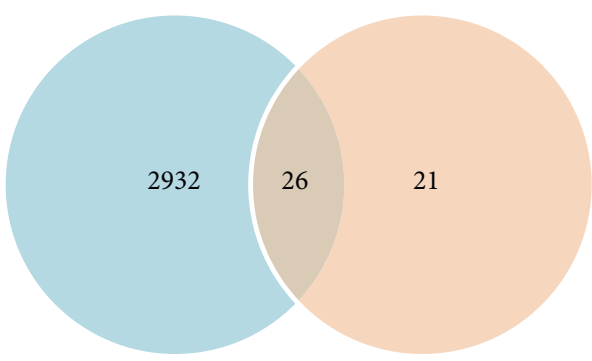

DB group versus WT group

GGQL group versus DB group

(b)

Figure 3: (a) Heatmap for cluster analysis of differentially expressed genes. (b) Venn diagram of differentially expressed genes.

with an analytical balance. The tissues were grinded, and cold 2: 1 chloroform: methanol was added. After vortexing, the samples were stored overnight at $4^{\circ} \mathrm{C}$. After centrifugation, the organic phase was extracted and dried under nitrogen. The samples were reconstituted for UPLC/MS/MS analysis. The content of ceramide was measured with a UPLC/MS/MS. Ceramide were analyzed using an Agilent TQ-S triple quadrupole mass spectrometer with positive ion electrospray ionization (ESI) source by Daughters Scan. The chromatographic separation was performed by a Waters UPLC Acquity. A reverse-phase Acquity BEH C18 column $(2.1 \times 100 \mathrm{~mm}, 1.7 \mu \mathrm{m})$ was used as the analytical column. Chromatographic separation was carried out in binary gradient with $2 \mathrm{mM}$ ammonium formate and $0.2 \%$ formic acid in water as solvent $\mathrm{A}$ and $1 \mathrm{mM}$ ammonium formate and $0.2 \%$ formic acid in methanol as solvent $\mathrm{B}$, and the flow rate was $0.4 \mathrm{ml} / \mathrm{min}$. The column temperature was $40^{\circ} \mathrm{C}$, and the injection volume was $2 \mu \mathrm{l}$.

2.8. Measurement of Pyruvate in Myocardial Tissue. The myocardial tissues were weighed about $20 \mathrm{mg}$ with an analytical balance. The protein concentration and pyruvate content of each tissue homogenate were measured according to the test kits.

2.9. Statistical Analysis. Differential expression analysis between each two groups was measured by using the DESeq $\mathrm{R}$ package (1.10.1), which provides statistical routines to determine differential expression in digital gene expression data using a model based on the negative binomial distribution. The $P$ values were adjusted with the Benjamini and Hochberg's approach for controlling the false discovery rate (FDR). Genes with an adjusted $P$ value $<0.05$ were considered to be differentially expressed.

All the data were presented as mean \pm SEM. Statistical analysis was performed with one-way analysis of variance (ANOVA) by SPSS program package (version 17.0). It is considered to be statistical significant when $P$ value was below 0.05 .

\section{Results}

3.1. Blood Glucose and Bodyweight. The results showed that the blood glucose and bodyweight of $\mathrm{db} / \mathrm{db}$ mice were significantly higher than those of WT mice. Gegen Qinlian 
TABLE 2: Genes significantly regulated in the GGQL group versus the DB group and also significantly regulated in the DB group versus the WT group.

\begin{tabular}{|c|c|c|c|}
\hline Gene name & Description & $\begin{array}{c}\text { Adjusted } P \text { value } \\
(\mathrm{DB} / \mathrm{WT})\end{array}$ & $\begin{array}{c}\text { Adjusted } P \text { value } \\
(\text { GGQL/DB) }\end{array}$ \\
\hline Pgam1 & Phosphoglycerate mutase 1 & $<0.0001$ & 0.0038 \\
\hline Igf1 & Insulin-like growth factor 1 & 0.0060 & 0.0029 \\
\hline Cpeb4 & Cytoplasmic polyadenylation element binding protein 4 & 0.0141 & 0.0283 \\
\hline Slc38a2 & Solute carrier family 38, member 2 & 0.0000 & $<0.0001$ \\
\hline Vegfa & Vascular endothelial growth factor A & 0.0001 & 0.0005 \\
\hline Banp & BTG3-associated nuclear protein & $<0.0001$ & 0.0100 \\
\hline Rbm38 & RNA-binding motif protein 38 & 0.0078 & 0.0380 \\
\hline Rasgef1b & RasGEF domain family, member $1 \mathrm{~B}$ & 0.0003 & 0.0037 \\
\hline Anxa3 & Annexin A3 & $<0.0001$ & 0.0309 \\
\hline Cypla1 & Cytochrome P450, family 1 , subfamily a, polypeptide 1 & $<0.0001$ & 0.0016 \\
\hline Trib1 & Tribbles homolog 1 (Drosophila) & 0.0004 & 0.0118 \\
\hline Nphp3 & Nephronophthisis 3 (adolescent) & $<0.0001$ & 0.0275 \\
\hline Fhl3 & Four and a half LIM domains 3 & 0.0036 & 0.0045 \\
\hline Serpine1 & Serine (or cysteine) peptidase inhibitor, clade E, member 1 & $<0.0001$ & 0.0016 \\
\hline Acer2 & Alkaline ceramidase 2 & $<0.0001$ & 0.0016 \\
\hline Arhgap32 & Rho GTPase activating protein 32 & $<0.0001$ & $<0.0001$ \\
\hline Atf 4 & Activating transcription factor 4 & 0.0044 & 0.0016 \\
\hline Zc3h6 & Zinc finger $\mathrm{CCCH}$ type containing 6 & $<0.0001$ & 0.0136 \\
\hline Tet1 & Tet methylcytosine dioxygenase 1 & 0.0006 & 0.0016 \\
\hline Klk1b26 & Kallikrein 1-related petidase b26 & 0.0146 & 0.0189 \\
\hline Ppp1r3c & Protein phosphatase 1 , regulatory (inhibitor) subunit $3 \mathrm{C}$ & 0.0000 & 0.0029 \\
\hline Ttc30b & Tetratricopeptide repeat domain $30 \mathrm{~B}$ & $<0.0001$ & 0.0054 \\
\hline Fign & Fidgetin & $<0.0001$ & 0.0195 \\
\hline AI593442 & Expressed sequence AI593442 & $<0.0001$ & 0.0350 \\
\hline 5730480H06Rik & RIKEN cDNA 5730480H06 gene & 0.0019 & 0.0029 \\
\hline Gm26703 & Predicted gene, 26703 & $<0.0001$ & $<0.0001$ \\
\hline
\end{tabular}

Genes with an adjusted $P$ value $<0.05$ were considered to be differentially expressed.

decoction could significantly reduce the blood glucose of the diabetic mice, but it had no significant effect on the bodyweight of the diabetic mice (Figure 1).

3.2. Effect of GGQL Decoction on Cardiac Function. The results of echocardiography showed that $A$ wave of the $\mathrm{DB}$ group increased significantly, and $E / A$ ratio and $E / A$ ' ratio decreased markedly compared to the WT group. The results indicated that the diastolic function of the left ventricle was impaired. After 8 weeks of treatment, compared to the DB group, $E / A$ ratio and $E / A$ ' ratio were recovered, and $A$ wave was also downregulated, suggesting that GGQL decoction could improve the diastolic dysfunction caused by diabetes mellitus. In three groups, there was no significant difference in $E$ wave (Figure 2).

3.3. Gene Expression Levels and DEGs. 15 samples from the WT group, DB group, and GGQL group were detected for DEGs, with 5 samples in each group. The hierarchal clustering results showed that 2958 genes were detected to be differentially expressed when comparing the DB group with the WT group, including 1450 upregulated genes and 1508 downregulated genes. Compared to the DB group, 47 genes were differentially regulated in the GGQL group (Figure 3). Among them, 26 genes were also differentially regulated in the DB group versus the WT group (Table 2), and 21 genes expressed no difference in the DB group versus the WT group (Table 3). The genes differentially expressed were related to anion binding, oxidoreductase activity, calcium-dependent phospholipid binding, ceramide metabolic process, and others.

3.4. Validation of Differentially Expressed Genes. Four differentially expressed genes were selected to validate by RT-qPCR, including Acer2, Slc38a2, Ppp1r3c, and Pgam1. Acer2 (alkaline ceramidase 2) regulates the hydrolysis of ceramides. Slc38a2 (solute carrier family 38 member 2) is a sodium-dependent amino acid transporter. Ppp1r3c (protein phosphatase 1 regulatory subunit $3 \mathrm{C}$ ) is a glycogen targeting subunit of PP1 and regulates glycogen metabolism. Pgam1 (phosphoglycerate mutase 1) is an enzyme which catalyzes the reaction of 3-phosphoglycerate (3-PGA) to 2-phosphoglycerate (2-PGA). 
TABLE 3: Genes significantly regulated in the GGQL group versus the DB group but no differential expression in the DB group versus the WT group.

\begin{tabular}{|c|c|c|c|}
\hline Gene name & Description & $\begin{array}{c}\text { Adjusted } P \text { value } \\
(\mathrm{DB} / \mathrm{WT})\end{array}$ & $\begin{array}{c}\text { Adjusted } P \text { value } \\
(\text { GGQL/DB) }\end{array}$ \\
\hline Gatsl3 & GATS protein-like 3 & 0.4265 & $<0.0001$ \\
\hline Abca8b & ATP-binding cassette, subfamily A (ABC1), member $8 \mathrm{~b}$ & 1 & 0.0195 \\
\hline Apex2 & Apurinic/apyrimidinic endonuclease 2 & 0.8103 & 0.0077 \\
\hline Dnajb4 & DnaJ (Hsp40) homolog, subfamily B, member 4 & 0.1556 & 0.0071 \\
\hline Vgll4 & Vestigial-like 4 (Drosophila) & 0.9649 & 0.0136 \\
\hline Klhl36 & Kelch-like 36 & 0.1950 & 0.0136 \\
\hline Amotl2 & Angiomotin-like 2 & 0.0637 & 0.0030 \\
\hline Cish & Cytokine inducible $\mathrm{SH} 2$-containing protein & 0.4114 & $<0.0001$ \\
\hline Myo6 & Myosin VI & 1 & 0.0483 \\
\hline Caskin2 & CASK-interacting protein 2 & 0.8910 & 0.0158 \\
\hline Bazla & Bromodomain adjacent to zinc finger domain $1 \mathrm{~A}$ & 0.2177 & 0.0020 \\
\hline Fbxw17 & F-box and WD-40 domain protein 17 & 0.6014 & 0.0480 \\
\hline Ddx60 & DEAD (Asp-Glu-Ala-Asp) box polypeptide 60 & 0.9151 & 0.0097 \\
\hline Gck & Glucokinase & 0.1227 & 0.0112 \\
\hline Zfp507 & Zinc finger protein 507 & 0.6355 & 0.0201 \\
\hline Zfp518a & Zinc finger protein $518 \mathrm{~A}$ & 1 & 0.0416 \\
\hline Dusp7 & Dual specificity phosphatase 7 & 0.7537 & 0.0416 \\
\hline Ifnlr1 & Interferon lambda receptor 1 & 0.3676 & 0.0275 \\
\hline Rnasel & Ribonuclease L ( $2^{\prime}, 5^{\prime}$-oligoisoadenylate synthetase-dependent) & 1 & 0.0275 \\
\hline Zfp763 & Zinc finger protein 763 & 0.2005 & 0.0063 \\
\hline RP23-402K24.5 & RIKEN cDNA 6230415J03 gene & 0.4670 & 0.0350 \\
\hline
\end{tabular}

Genes with an adjusted $P$ value $<0.05$ were considered to be differentially expressed.

The results of RNA-seq showed that, compared with the WT group, the expressions of Acer2, Slc38a2, Ppp1r3c, and Pgam1 were depressed in LV tissue of the DB group mice. And the expressions of all the four genes were significantly upregulated in the GGQL group. The results of RT-qPCR were consistent with the results of RNA-seq, and the results of RNA-seq were validated (Figure 4).

3.5. Content of Ceramide in Myocardial Tissue. The content of ceramide (d18:1/24:1) in the myocardial tissue of the model group was markedly higher than that of the wildtype group $(P<0.05)$. In the model group, the content of ceramide (d18:1/24:0) and ceramide (d17:1/24:1) appeared an increasing trend compared to that of the wild-type group. The levels of ceramide (d18:1/24:1), ceramide (d18:1/24:0), and ceramide $(\mathrm{d} 17: 1 / 24: 1)$ in the myocardial tissue of the GGQL group had a reduced trend compared to that of the model group (Figure 5).

3.6. Content of Pyruvate in Myocardial Tissue. In the model group, the pyruvate content of myocardial tissue homogenate decreased significantly than that of the wild-type group $(P<0.05)$. Compared to the model group, the content of pyruvate in the myocardial tissue homogenate of the GGQL group increased obviously $(P<0.05)$ (Figure 5).

\section{Discussion}

DM is not only manifested as long-term and chronic elevated hyperglycemia but also accompanied with various complications. Diabetic cardiomyopathy (DCM) is one of the major causes of death in T2DM patients. DCM is a type of primary cardiomyopathy, independent of macrovascular and coronary artery diseases. The pathophysiology of DCM includes cardiomyocyte hypertrophy, focal necrosis, extracellular matrix accumulation, and interstitial fibrosis [12]. Early manifestations usually expressed as diastolic dysfunction with major pathological changes of declining in myocardial compliance and blocked diastolic filling [13]. Systolic dysfunction is the major pathological change of DCM in the late period of the disease, and this disturbance is prone to congestive heart failure.

$\mathrm{db} / \mathrm{db}$ mice are the classic models for the research of T2DM-induced DCM. A number of investigators confirmed the existence of DCM of $\mathrm{db} / \mathrm{db}$ mice with evaluation of echocardiography $[14,15]$. According to the literature, under the detection of echocardiography, the $E / A$ ratio and $E$ ' $/ A$ ' ratio of the $\mathrm{db} / \mathrm{db}$ mice decreased significantly compared with WT mice $[14,16,17]$, suggesting impaired cardiac diastolic function.

In our study, compared with the WT mice, the $A$ wave increased, and the left ventricular $E / A$ and $E^{\prime} / A^{\prime}$ ratio of the 


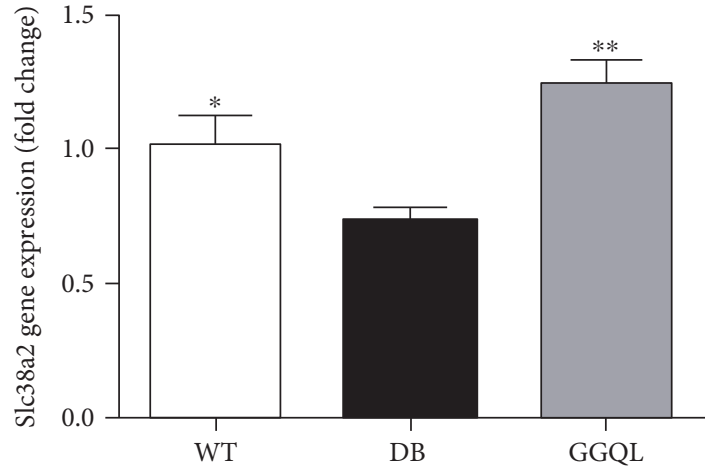

(a)

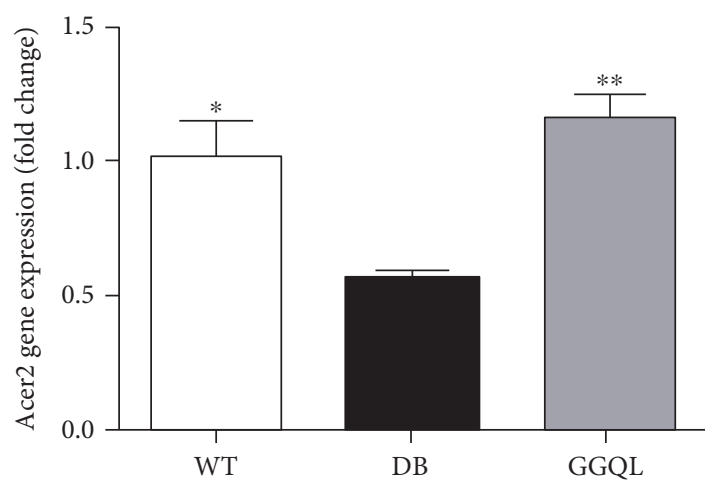

(c)

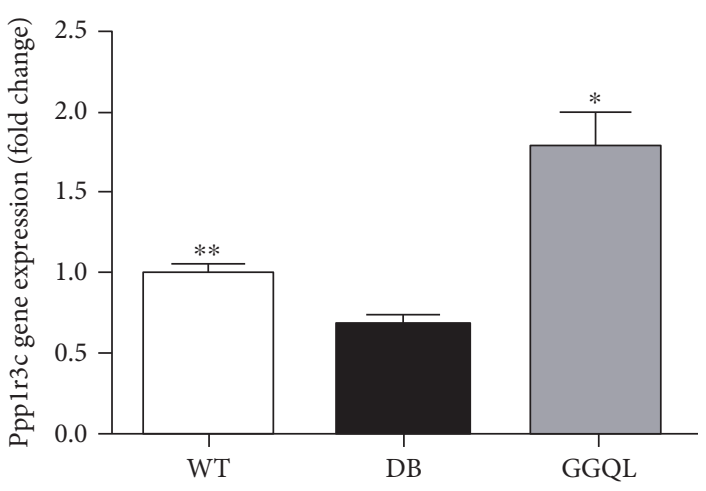

(b)

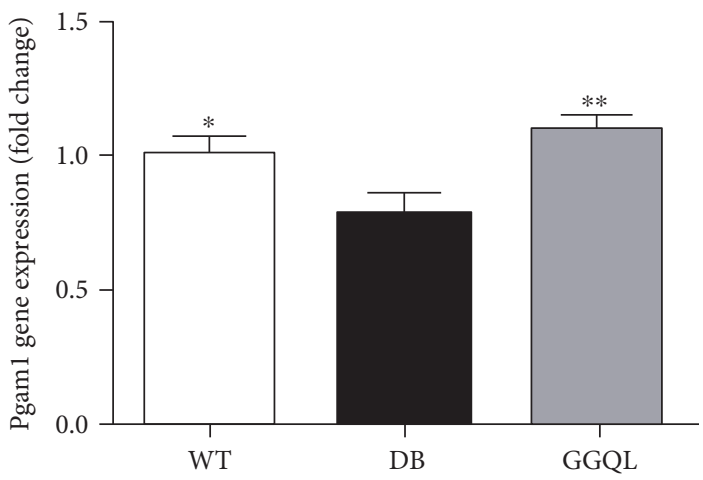

(d)

Figure 4: The results of RT-qPCR to validate differentially expressed genes. The mRNA expressions of (a) Slc38a2, (b) Ppp1r3c, (c) Acer2, and (d) Pgam1 in each group $(n=4-5)$. The DB group was the reference group to calculate $P$ values, ${ }^{*} P<0.05$ and ${ }^{* *} P<0.01$.

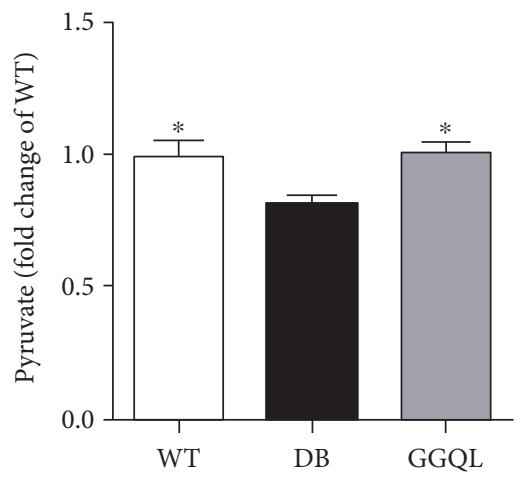

(a)

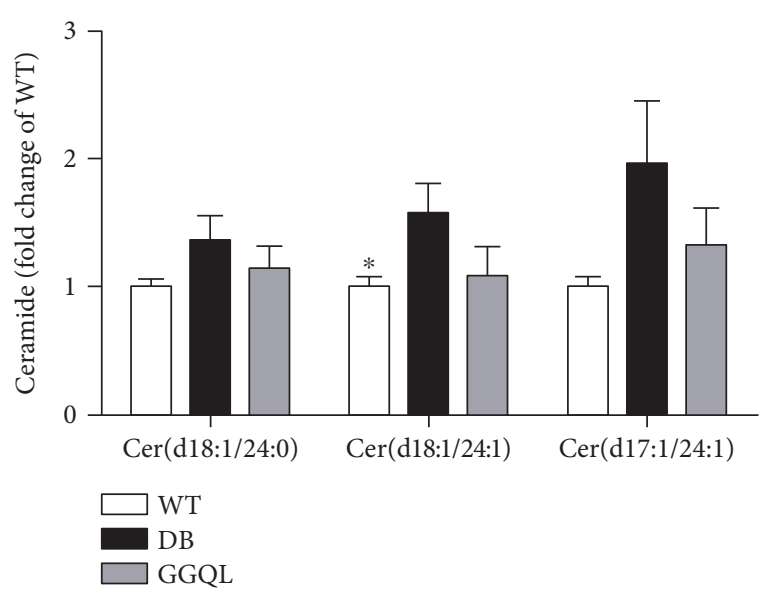

(b)

FIgURE 5: The results of pyruvate and ceramide content in the myocardial tissue. The content of (a) pyruvate and (b) ceramide in each group $(n=3-6)$. The DB group was the reference group to calculate $P$ values, ${ }^{*} P<0.05$.

$\mathrm{db} / \mathrm{db}$ mice markedly decreased, suggesting a diastolic dysfunction of the left ventricular; Gegen Qinlian decoction could significantly increase the left ventricle $E / A$ and $E / A^{\prime}$ ratio of the $\mathrm{db} / \mathrm{db}$ mice and downregulate the $A$ wave, suggesting that the drug can improve the function of the left ventricle.
Glycolysis is a process in which one molecule of glucose is converted to two molecules of pyruvate and produces two molecules of adenosine triphosphate (ATP), which provides energy for almost all the biological processes. Glycolysis is necessary for cardiac metabolism. Glucose utilization is impaired, meanwhile glycolysis and glucose oxidation are 
depressed in DCM. In diabetes, the capacity of cardiac glycolysis is chronically reduced [18]. The reduction of myocardial glycolysis may be associated with the development of heart failure and myocardial injury suffered by DM patients $[19,20]$.

Glycolysis consists of ten steps, and pyruvate is the product. Phosphoglycerate mutase-1 (Pgam1) is used to catalyze the interconversion of 3-phosphoglycerate (3-PGA) to 2-phosphoglycerate (2-PGA), which is the eighth step of glycolysis [21].

Ceramide is a main molecule of the sphingolipid metabolism, which mediates cell growth retardation and differentiation, and inhibits cell proliferation and promotes apoptosis [22-26]. The accumulation of tissue ceramides may be helpful to the development of insulin resistance [27]. Ceramide is an important mediator of myocardial lipid toxicity $[28,29]$. Moreover, some studies showed that ceramide is related to cardiomyocyte apoptosis induced by ischemia/reperfusion injury [30, 31]. Alkaline ceramidase 2 (Acer2) is a type of ceramidases, which are key enzymes of the degradation of intracellular ceramide and hydrolyzed ceramide to sphingosine and then further phosphorylated to sphingosine-1-phosphate $(\mathrm{S} 1 \mathrm{P})$ by sphingosine kinase [32]. And the increased expression of Acer2 may cause the decrease in ceramide accumulation.

Previous research indicated that the cardiac glycolytic rate of $\mathrm{db} / \mathrm{db}$ mice was reduced. Cardiac function, glycolysis, and glucose oxidation of isolated hearts of C57BL/KsJ $(d b / d b)$ were damaged [19]. It is also reported that in the diabetic model animals, myocardial ceramide content was significantly increased accompanied with cardiac dysfunction $[33,34]$.

The results of this study showed that compared with the mice of the WT group, the mRNA content of Pgam1 and Acer2 in myocardium of the DB group mice decreased significantly. And the mRNA content of Pgam 1 and Acer2 in myocardium of the GGQL group mice increased significantly, compared with the mice of the DB group. It indicated that the myocardial glycolysis and ceramide hydrolysis were depressed in the DB group mice, and potentially the treatment of Gegen Qinlian decoction protects the myocardium by promoting glycolysis and decreasing the content of ceramide. In this study, the results of the pyruvate content in the myocardial tissue suggested that the glycolysis decreased in the DB group compared to the WT group. And GGQL decoction has the effect to increase cardiac glycolysis in diabetic mice. The content of ceramide in the myocardial tissue of the DB group appeared an increasing trend compared to that of the wild-type group, and GGQL decoction trended to reduce the ceramide content in diabetic mice. The results were consistent with the results of RNA-seq and q-PCR.

Previous studies have shown that berberine has protective effect on the development of diabetic cardiomyopathy [35]. Liquiritigenin and liquiritin have a protective role in high fructose-induced myocardial fibrosis [36, 37]. Other investigators confirmed that various components of Gegen Qinlian decoction have moderating effect on glycolysis and ceramide levels. Berberine could significantly reduce serum levels of ceramide of nonalcoholic fatty liver disease patients
[38] and stimulate the glycolysis in HepG2 hepatocytes and C2C12 myotubes [39]. Berberine compounds could upregulate the glycolysis which was depressed in hyperlipidemic rats [40]. Baicalin could decreased the blood glucose of diabetic rats by increasing the content of liver glycogen and promoting glycolysis [41]. It is concluded that the effect on improving diastolic function of the left ventricle and promoting the mRNA expression of Pgam1 and Acer2 of Gegen Qinlian decoction may be attributed to berberine, baicalin, liquiritigenin, and liquiritin.

In conclusion, the results of this study showed that Gegen Qinlian decoction has a therapeutic effect on diastolic dysfunction of the left ventricle in $\mathrm{db} / \mathrm{db}$ mice, and the effect may be related to its role in promoting myocardial glycolysis and decreasing the content of ceramide.

\section{Conflicts of Interest}

The authors declare that they have no competing interests.

\section{Authors' Contributions}

Yan Wu conceived and designed the experiments. Jing Han, Zhenglin Wang, Wei Xing, Yueying Yuan, and Yonggang Liu performed the experiments. Tiantian Lv, Hongliang Wang, and Yi Zhang analyzed the data. Jing Han and Yan Wu contributed to the writing of the manuscript. All authors read and approved the final manuscript.

\section{Acknowledgments}

This work was supported by the National Nature Science Foundation of China (no. 81303083).

\section{References}

[1] W. Chen, Y. Xia, X. Zhao et al., "The critical role of Astragalus polysaccharides for the improvement of PPAR $\alpha$-mediated lipotoxicity in diabetic cardiomyopathy," PLoS One, vol. 7, no. 10, article e45541, 2012.

[2] K. Trachanas, S. Sideris, C. Aggeli et al., "Diabetic cardiomyopathy: from pathophysiology to treatment," Hellenic Journal of Cardiology, vol. 55, no. 5, pp. 411-421, 2014.

[3] K. Huynh, H. Kiriazis, L. J. E. Du XJ et al., "Coenzyme $\mathrm{Q}_{10}$ attenuates diastolic dysfunction, cardiomyocyte hypertrophy and cardiac fibrosis in the $d b / d b$ mouse model of type 2 diabetes," Diabetologia, vol. 55, no. 5, pp. 1544-1553, 2012.

[4] J. Fuentes-Antras, B. Picatoste, E. Ramirez, J. Egido, J. Tunon, and O. Lorenzo, "Targeting metabolic disturbance in the diabetic heart," Cardiovascular Diabetology, vol. 14, no. 1, p. 17, 2015.

[5] Y. P. Zeng, Y. S. Huang, and Y. G. Hu, "Effect of Gegen Qinlian decoction combined with short-term intensive insulin treatment on patients with type 2 diabetes mellitus of dampnessheat syndrome," Zhongguo Zhong Xi Yi Jie He Za Zhi, vol. 26, no. 6, pp. 514-516, 2006, 520.

[6] Y. M. Li, X. M. Fan, Y. M. Wang, Q. L. Liang, and G. A. Luo, "Therapeutic effects of Gegen Qinlian decoction and its mechanism of action on type 2 diabetic rats," Yao Xue Xue Bao, vol. 48, no. 9, pp. 1415-1421, 2013. 
[7] J. Li, T. Gao, Q. Song, H. Li, Y. Wang, and Q. Feng, "Study on mechanism of effect of Gegenqinlian decoction on lowering blood sugar and lipid of type 2 diabetic rats," Hu Bei Zhong Yi Yao Da Xue Xue Bao, vol. 17, pp. 7-9, 2015.

[8] Y. Tan, Y. Shi, L. Liu, and Y. Zhao, "Effects of Gegenqinlian decoction and effective components on the isolated perfused heart," Journal of Harbin University of Commerce(Natural Sciences Edition), vol. 27, pp. 649-652, 2011.

[9] E. Chan, X. X. Liu, D. J. Guo et al., "Extract of Scutellaria baicalensis Georgi root exerts protection against myocardial ischemia-reperfusion injury in rats," The American Journal of Chinese Medicine, vol. 39, no. 4, pp. 693-704, 2011.

[10] C. W. Lau, X. Q. Yao, Z. Y. Chen, W. H. Ko, and Y. Huang, "Cardiovascular actions of berberine," Cardiovascular Drug Reviews, vol. 19, no. 3, pp. 234-244, 2001.

[11] Y. Wang, W. Lin, C. Li et al., "Multipronged therapeutic effects of Chinese herbal medicine Qishenyiqi in the treatment of acute myocardial infarction," Frontiers in Pharmacology, vol. 8, p. 98, 2017.

[12] R. B. Devereux, M. J. Roman, M. Paranicas et al., "Impact of diabetes on cardiac structure and function: the strong heart study," Circulation, vol. 101, no. 19, pp. 2271-2276, 2000.

[13] C. M. Schannwell, M. Schneppenheim, S. Perings, G. Plehn, and B. E. Strauer, "Left ventricular diastolic dysfunction as an early manifestation of diabetic cardiomyopathy," Cardiology, vol. 98, no. 1-2, pp. 33-39, 2002.

[14] L. M. Semeniuk, A. J. Kryski, and D. L. Severson, "Echocardiographic assessment of cardiac function in diabetic $d b / d b$ and transgenic $d b / d b$-hGLUT4 mice," American Journal of Physiology Heart and Circulatory Physiology, vol. 283, no. 3, pp. H976-H982, 2002.

[15] K. Venardos, K. A. De Jong, M. Elkamie, T. Connor, and S. L. McGee, "The PKD inhibitor CID755673 enhances cardiac function in diabetic $d b / d b$ mice," PLoS One, vol. 10, no. 3, article e0120934, 2015.

[16] M. van Bilsen, A. Daniels, O. Brouwers et al., "Hypertension is a conditional factor for the development of cardiac hypertrophy in type 2 diabetic mice," PLoS One, vol. 9, no. 1, article e85078, 2014.

[17] D. J. Stuckey, C. A. Carr, D. J. Tyler, E. Aasum, and K. Clarke, "Novel MRI method to detect altered left ventricular ejection and filling patterns in rodent models of disease," Magnetic Resonance in Medicine, vol. 60, no. 3, pp. 582-587, 2008.

[18] A. Avogaro, R. Nosadini, A. Doria et al., "Myocardial metabolism in insulin-deficient diabetic humans without coronary artery disease," The American Journal of Physiology, vol. 258, no. 4, Part 1, pp. E606-E618, 1990.

[19] D. D. Belke, T. S. Larsen, E. M. Gibbs, and D. L. Severson, "Altered metabolism causes cardiac dysfunction in perfused hearts from diabetic $(d b / d b)$ mice," American Journal of Physiology Endocrinology and Metabolism, vol. 279, no. 5, pp. E1104-E1113, 2000.

[20] E. D. Abel, H. C. Kaulbach, R. Tian et al., "Cardiac hypertrophy with preserved contractile function after selective deletion of GLUT4 from the heart," Journal of Clinical Investigation, vol. 104, no. 12, pp. 1703-1714, 1999.

[21] L. A. Fothergill-Gilmore and H. C. Watson, "The phosphoglycerate mutases," Advances in Enzymology and Related Areas of Molecular Biology, vol. 62, pp. 227-313, 1989.
[22] Y. Mizutani, H. Sun, Y. Ohno et al., "Cooperative synthesis of ultra long-chain fatty acid and ceramide during keratinocyte differentiation," PLoS One, vol. 8, no. 6, article e67317, 2013.

[23] B. J. Pettus, C. E. Chalfant, and Y. A. Hannun, "Ceramide in apoptosis: an overview and current perspectives," Biochimica et Biophysica Acta (BBA) - Molecular and Cell Biology of Lipids, vol. 1585, no. 2-3, pp. 114-125, 2002.

[24] T. A. Taha, T. D. Mullen, and L. M. Obeid, "A house divided: ceramide, sphingosine, and sphingosine-1-phosphate in programmed cell death," Biochimica et Biophysica Acta (BBA) Biomembranes, vol. 1758, no. 12, pp. 2027-2036, 2006.

[25] S. Furuya, J. Mitoma, A. Makino, and Y. Hirabayashi, "Ceramide and its interconvertible metabolite sphingosine function as indispensable lipid factors involved in survival and dendritic differentiation of cerebellar Purkinje cells," Journal of Neurochemistry, vol. 71, no. 1, pp. 366-377, 1998.

[26] Y. Uchida, A. D. Nardo, V. Collins, P. M. Elias, and W. M. Holleran, "De novo ceramide synthesis participates in the ultraviolet B irradiation-induced apoptosis in undifferentiated cultured human keratinocytes," Journal of Investigative Dermatology, vol. 120, no. 4, pp. 662-669, 2003.

[27] S. A. Summers, "Ceramides in insulin resistance and lipotoxicity," Progress in Lipid Research, vol. 45, no. 1, pp. 42-72, 2006.

[28] H. C. Chiu, A. Kovacs, D. A. Ford et al., "A novel mouse model of lipotoxic cardiomyopathy," Journal of Clinical Investigation, vol. 107, no. 7, pp. 813-822, 2001.

[29] D. L. Hickson-Bick, L. M. Buja, and J. B. McMillin, "Palmitate-mediated alterations in the fatty acid metabolism of rat neonatal cardiac myocytes," Journal of Molecular and Cellular Cardiology, vol. 32, no. 3, pp. 511-519, 2000.

[30] A. E. Bielawska, J. P. Shapiro, L. Jiang et al., "Ceramide is involved in triggering of cardiomyocyte apoptosis induced by ischemia and reperfusion," American Journal of Pathology, vol. 151, no. 5, pp. 1257-1263, 1997.

[31] G. A. Cordis, T. Yoshida, and D. K. Das, "HPTLC analysis of sphingomylein, ceramide and sphingosine in ischemic/reperfused rat heart," Journal of Pharmaceutical and Biomedical Analysis, vol. 16, no. 7, pp. 1189-1193, 1998.

[32] P. W. Wertz and D. T. Downing, "Ceramidase activity in porcine epidermis," FEBS Letters, vol. 268, no. 1, pp. 110-112, 1990.

[33] Y. T. Zhou, P. Grayburn, A. Karim et al., "Lipotoxic heart disease in obese rats: implications for human obesity," Proceedings of the National Academy of Sciences of the United States of America, vol. 97, no. 4, pp. 1784-1789, 2000.

[34] R. Basu, G. Y. Oudit, X. Wang et al., "Type 1 diabetic cardiomyopathy in the Akita (Ins2WT/C96Y) mouse model is characterized by lipotoxicity and diastolic dysfunction with preserved systolic function," American Journal of Physiology. Heart and Circulatory Physiology, vol. 297, no. 6, pp. H2096H2108, 2009.

[35] W. Chang, M. Zhang, Z. Meng et al., "Berberine treatment prevents cardiac dysfunction and remodeling through activation of $5^{\prime}$-adenosine monophosphate-activated protein kinase in type 2 diabetic rats and in palmitate-induced hypertrophic H9c2 cells," European Journal of Pharmacology, vol. 769, pp. 55-63, 2015.

[36] Y. Zhang, L. Zhang, Y. Zhang, J. J. Xu, L. L. Sun, and S. Z. Li, "The protective role of liquiritin in high fructose-induced myocardial fibrosis via inhibiting NF- $\kappa \mathrm{B}$ and MAPK signaling pathway," Biomedicine \& Pharmacotherapy, vol. 84, pp. 13371349, 2016. 
[37] X. W. Xie, "Liquiritigenin attenuates cardiac injury induced by high fructose-feeding through fibrosis and inflammation suppression," Biomedicine \& Pharmacotherapy, vol. 86, pp. 694-704, 2017.

[38] X. Chang, Z. Wang, J. Zhang et al., "Lipid profiling of the therapeutic effects of berberine in patients with nonalcoholic fatty liver disease," Journal of Translational Medicine, vol. 14, no. 1, p. 266, 2016.

[39] M. Xu, Y. Xiao, J. Yin et al., "Berberine promotes glucose consumption independently of AMP-activated protein kinase activation," PLoS One, vol. 9, no. 7, article e103702, 2014.

[40] M. Li, X. Shu, H. Xu et al., "Integrative analysis of metabolome and gut microbiota in diet-induced hyperlipidemic rats treated with berberine compounds," Journal of Translational Medicine, vol. 14, no. 1, p. 237, 2016.

[41] H. T. Li, X. D. Wu, A. K. Davey, and J. Wang, "Antihyperglycemic effects of baicalin on streptozotocin - nicotinamide induced diabetic rats," Phytotherapy Research, vol. 25, no. 2, pp. 189-194, 2011. 

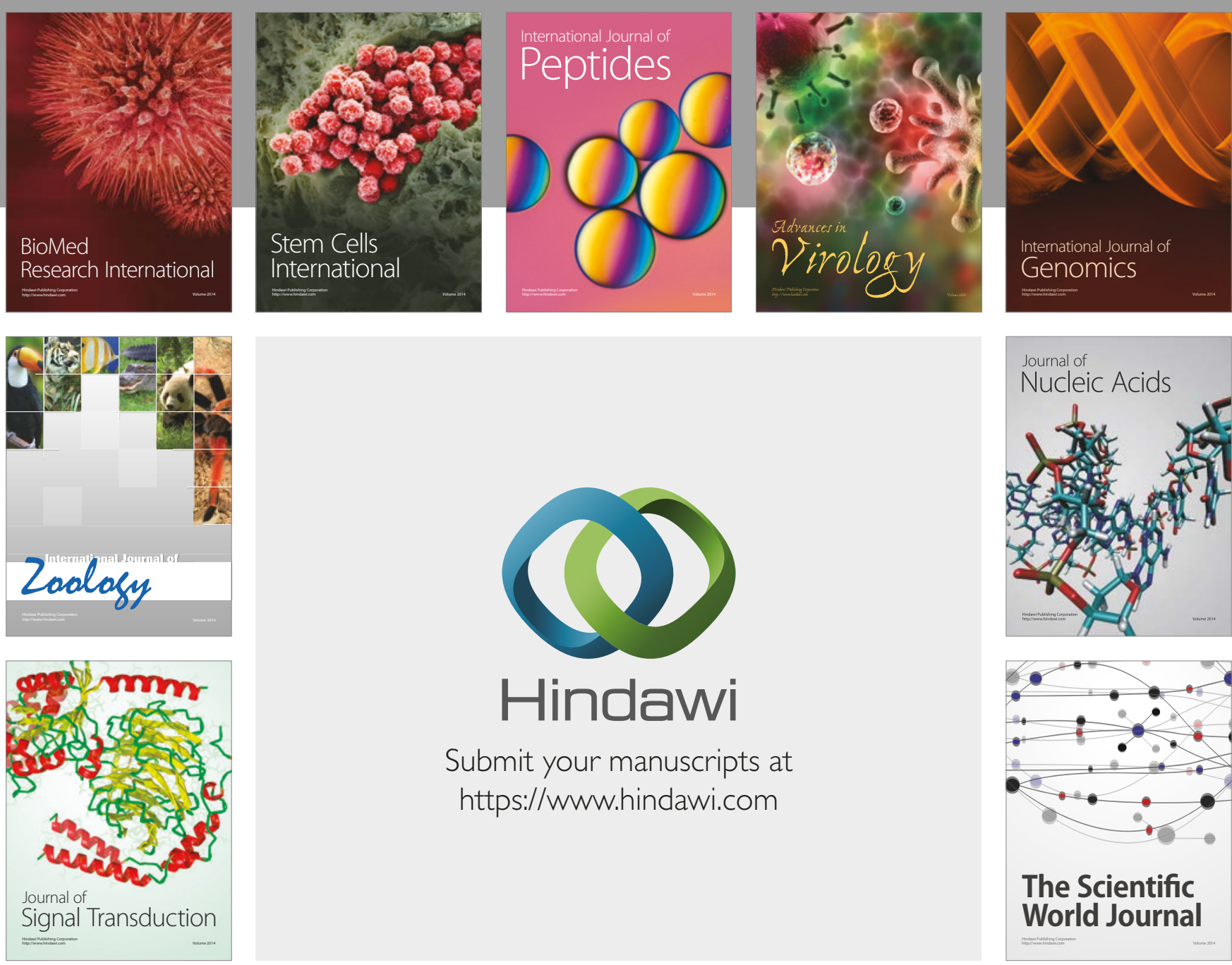

Submit your manuscripts at

https://www.hindawi.com
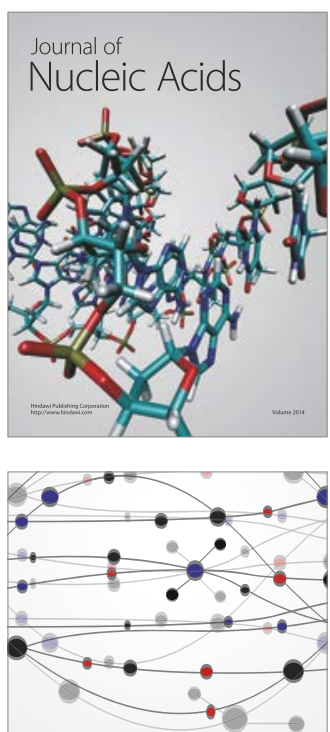

The Scientific World Journal

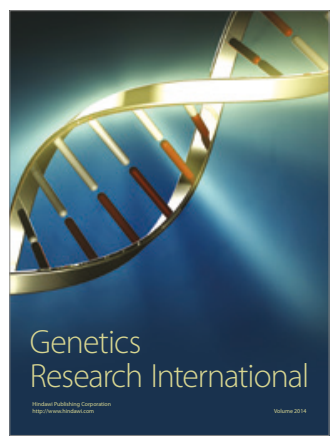

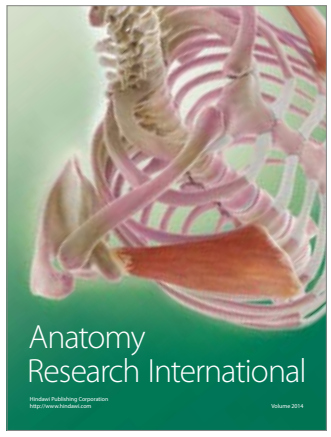

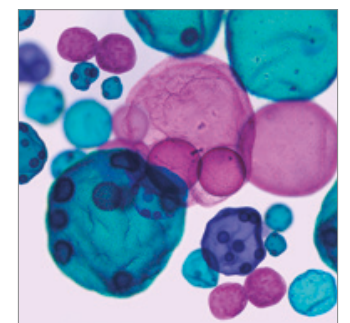

International Journal of Microbiology
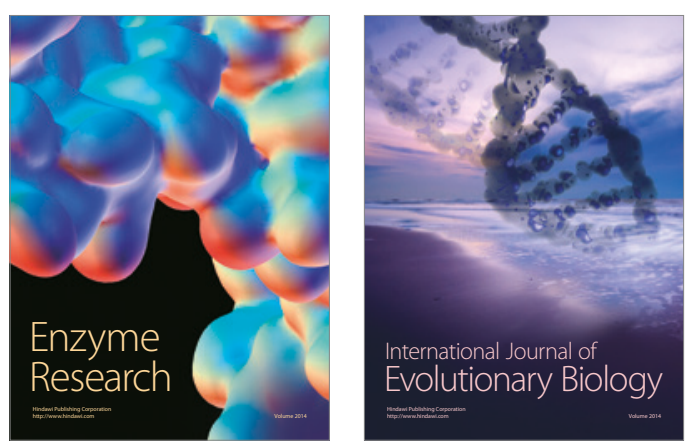
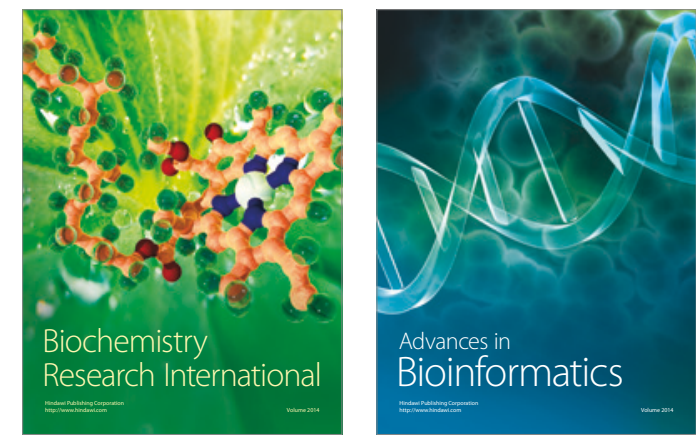

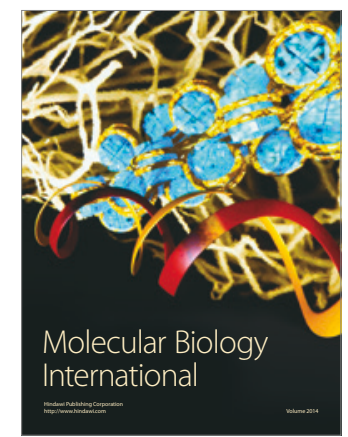

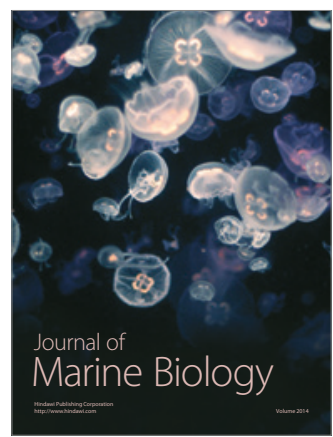

